\title{
The use of morselized allografts without impaction and cemented cage support in acetabular revision surgery: a 4- to 9-year follow-up
}

Huifeng Ding ${ }^{1,2 \dagger}$, Yuanqing Mao ${ }^{1 \dagger}$, Bin $\mathrm{Yu}^{2 \dagger}$, Zhenan Zhu ${ }^{1 *}$, Huiwu $\mathrm{Li}^{1}$, Baoqing $\mathrm{Yu}^{2^{*}}$ and Jianming Huang ${ }^{2}$

\begin{abstract}
Background: Acetabular revision arthroplasty with major bone loss is one of the most difficult operations in orthopedic surgery. The goal of the study was to evaluate midterm clinical results of the use of morselized allografts with cemented cage support in revision total hip replacement.
\end{abstract}

Methods: We identified 28 patients (29 hips) at an average follow-up of 73 months. Harris Hip Scores (HHS) were assessed before and after surgery. Pre- and postoperative radiographs were evaluated for restoration of the center of rotation, component migration, and graft incorporation.

Results and discussion: At follow-up, the mean HHS improved from 34 (range, 20-45) to 80 (range, 71-98) points. None of the components had been re-revised. On average, the revised hip center of rotation was improved significantly. Incorporation of the graft was complete in 23 hips. The midterm result of cage reconstruction with morselized bone allograft is relatively better than other studies using a similar cage construction. We believe we have three special modifications of this reconstruction technique that are beneficial for bone incorporation.

Conclusions: These data confirm that acetabular reconstruction using morselized allografts and cemented acetabular cages is effective in the midterm as a treatment for acetabular loosening with massive bone deficiency.

Keywords: Acetabulum, Prosthesis, Failure, Revision, Allograft, Cage

\section{Introduction}

Acetabular revision arthroplasty with major bone loss is one of the most difficult operations in orthopedic surgery. The primary goal of surgery is to obtain a stable, durable reconstruction. Secondary goals include reconstituting bone stock, restoring the hip center of rotation to the anatomical location, and minimizing leg-length discrepancies. To meet these goals, the use of bone graft and reconstruction cage devices in acetabular revisions has become the popular surgery for this form of major bone loss.

Reconstruction cages spanning from the ilium to the ischium have been used with morselized allografts to

\footnotetext{
*Correspondence: zhuzhenan2010@126.com; doctorybq@163.com ${ }^{\dagger}$ Equal contributors

'Shanghai Key Laboratory of Orthopaedic Implants, Department of Orthopaedics, Ninth People's Hospital, Shanghai Jiao Tong University School of Medicine, Shanghai, The People's Republic of China

${ }^{2}$ Department of Orthopaedics, Shanghai Pudong Hospital, Fudan University Pudong Medical Center, Shanghai, The People's Republic of China
}

revise massive acetabular deficiencies. Success rates for the use of these cages with morselized bone to reconstruct massive acetabular defects have ranged from 41 to $100 \%$ at intermediate-term follow-up [1-5]. There are few papers focused on failures in cage construction. Böhm and Banzhaf [6] identified three factors that correlated with failure: lack of radiographic incorporation of the allograft, graft resorption, and the use of a particulate (morselized) as opposed to a bulk (structural) allograft. In contrast, Sembrano and Cheng [7] concluded that no single preoperative factor (age, gender, severity of pelvic defect, degree of heterotopic ossification, difference in limb lengths, or center of rotation) or intraoperative factor (type of bone graft, type of cage, changes in limb length, or center of rotation) predicted cage failure.

But most studies report that the failure of revision is caused by a failure of bone integration. A reconstruction composed of the host bone, an allograft, and an implant will be successful only if the entire compound remains 
stable. Böhm and Banzhaf [6] have postulated that the various sources of failure are related to two biomechanical situations: insufficient primary stability or poor incorporation of the allograft into the host bone. Sun et al. [8] proposed that the incorporation of the allograft is a vital factor for the success of acetabular cup revision. The primary cause of cup radiographic failures in his study was the failure of the allograft incorporation.

With the use of an acetabular reconstruction cage, primary stability is not difficult to achieve. Thus, we focused more attention on the incorporation of the allograft to the host bone. The purpose of our retrospective study is to review data from our 4- to 9-year follow-up evaluation of complex acetabular revisions with morselized bone allografts and cemented cage supports. Our goals are to achieve excellent primary stability and to perfect allograft incorporation during and after the operation.

\section{Patients and methods Patients}

This study was approved by the ethics committee of Shanghai Ninth People's Hospital and Shanghai Pudong Hospital. Written informed consents were obtained from all participants.

We treated 30 patients with morselized allograft and acetabular reinforcement cages at our institution between 2005 and 2010. The patients were followed up for a minimum of 4 years. Two patients were lost due to failure to follow up, leaving 28 patients (29 hips) for review. The overall follow-up averaged 73 months (range, 48-108).

The average age at primary hip arthroplasty was 43 years (range, 25-60). The average age at acetabular revision was 61 years (range, 43-75). The diagnosis leading to the original THA was osteoarthritis in seven hips, femoral neck fracture in six hips, developmental dysplasia in four hips, ankylosing spondylitis in three hips, rheumatoid arthritis in three hips, avascular necrosis in five hips, and a bone tumor in one hip. The original acetabular arthroplasty was uncemented in 16 hips and cemented in 10 hips, and bipolar hemiarthroplasty was performed for 3 hips. The reason for failure and the need for subsequent revision of all 29 hips was aseptic loosening. The average number of prior hip operations was 1.4 (range, 1-3). The type and extent of the acetabular deficiency was determined from preoperative radiographs and confirmed by intraoperative findings. The deficiencies were classified according to the system devised by Paprosky et al. [9]. Depending on the roentgenographic and intraoperative findings on the acetabulum, the acetabular deficiency was classified according to the classification system of Paprosky classification [9]: 2B, 3 hips (10\%); 2C, 2 hips (7\%); 3A, 14 hips (48\%); and 3B, 10 hips (35\%). No patients had pelvic discontinuity.

\section{Clinical and radiological assessments}

The results were analyzed with respect to both clinical and radiographic parameters. Harris Hip Scores (HHS) [10] were obtained both preoperatively and at the latest follow-up. Hips with scores ranging from 90 to 100 points were graded as having excellent results, hips with scores ranging from 80 to 89 points were graded as having good results, those hips with scores ranging from 70 to 79 points were graded as having fair results, and those with scores $<70$ points were graded as having poor results.

The radiographs were measured preoperatively, immediately postoperatively, and at final follow-up, similar to the method described by Peters et al. [11]. Two lines were drawn: a horizontal inter-teardrop reference line and a perpendicular reference line through the teardrop. When the teardrop was destroyed, preoperative roentgenograms with an intact teardrop or an opposite teardrop were adopted as a symmetric reference point. We measured the changes in distance between the center of hip rotation and two reference lines. Vertical distance was defined as the distance between the center of hip rotation and horizontal inter-teardrop reference line. Horizontal distance was defined as the distance between the center of hip rotation and perpendicular reference line. The magnification of radiographs was corrected by the known diameter of the metal femoral head. Significant migration was defined as a change in the acetabular inclination of $5^{\circ}$ or more or linear migration of the hip center of $5 \mathrm{~mm}$ or more.

Bone-implant radiolucent lines around the cage and screws were measured in millimeters on the anteroposterior radiographs according to the zonal analysis of DeLee and Charnley [12]. The stability of the acetabular implant was assessed according to the criteria of Gill et al. [13] and Van der Linde and Tonino [14]: definitely loose (screw breakage or acetabular migration $>5 \mathrm{~mm}$ or progressive radiolucent lines present at the cage-bone interface medially and superiorly or around the screws), most likely loose (progressive radiolucencies present medially or superiorly), and possibly loose (radiolucencies are nonprogressive and do not involve the screws). Van der Linde and Tonino [14] stated that breakage of the screws without continuing migration or change in inclination of the cage should not be defined as failure. Allograft bone was assessed radiologically for union as evidenced by trabecular bridging at the donor-host interface as previously defined $[8,11,15,16]$.

\section{Implantation and technique}

All revision procedures were performed by one senior surgeon. The acetabular component consisted of a cemented hemispherical titanium alloy cup, an acetabular hook, and three iliac flanges (revision cup system, Lima corporate spa, Udine, Italy). Although this cage is 
first-generation production from Lima corporate spa, it is still used popularly in China because most people could not afford the second-generation production although it is more advanced because of a snap-in locking mechanism of the liner [17]. A posterolateral approach was used in all hips. A trochanteric osteotomy was performed in six hips to expose the acetabulum and the outer surface of the ilium. After exposure of the entire acetabulum, the acetabular component, the cement, and underlying fibrous membrane were carefully removed. The acetabulum was reamed with progressively larger reamers until a bed of sclerotic bone appeared. When we prepared the bone bed for the graft bone, we always induced bleeding in the sclerotic bone using the K-wire or bone knife, as we thought this would improve the revascularization of the sclerotic bone and graft bone. Cage size was provisionally decided upon from the size of the last reamer and was confirmed with a trial cup. The contact and stability of the trial cage against supportive host bone were then assessed, and the need for the use of a reconstruction cage was determined. Two to four heads of morselized, fresh-frozen femoral head allograft from the bone bank (cancellous-cortical allograft produced using a bone mill) were positioned into the cleared acetabulum and were reversely reamed. We did not impact the allograft bone very tightly with an impactor. The acetabulum was then shaped into a hemispherical form using the acetabular reamer, and the appropriate acetabular cage size was chosen. We selected the reconstruction cage according to the size of acetabular bone defect. The best choice of cage was considered to be that which was large enough to directly contact the host bone at the upper side, where the inferior hook could be tightly affixed to the teardrop. If the cage was not large enough, we preferred to place the cage in direct contact with the host bone at the upper side because the contact between the cage and host bone could provide more primary stability than the hook. When the hook of the device was inserted under the teardrop, the acetabular cup was pushed into the prepared acetabulum. Iliac flanges were configured on the outer surface of the ilium to obtain the maximum contact between the flanges and the iliac bone. If there was still a gap between the flanges and the iliac bone, we filled the gap around the flange with morselized allograft. The iliac flanges were then fixed with screws. It was also important that the screw was not affixed vertically to the flange, as this could prevent cutting between the screw and the flange. The average cage size of the components was $58 \mathrm{~mm}$ (range, 54-64). The average number of total screws was 4.9 (range, 3-7). A $28-\mathrm{mm}$ head was used in all 29 hips. Morselized allograft bone was used to fill the defects. Bulk structural allograft was not used in all deficiencies.

\section{Postoperative care}

Physical therapy began on the first postoperative day. The patients were advised to avoid flexion of the affected hip joint beyond $90^{\circ}$ and to avoid forced internal rotation. Slight abduction was ensured for 2 weeks with the use of a wedged pillow. Partial weight bearing was allowed 6 weeks after surgery and continued for a duration of 4 to 6 weeks. After that, activities progressed toward full weight-bearing. Clinical and radiographic follow-up examinations were performed at 3 months, 6 months, and 1 year after the operation, and once a year thereafter. For patients with relatively large bone defects, especially at the lateral upper region of the acetabulum, we did not permit total weight-bearing at 3 months after the revision. Instead, we encouraged the patient to use a walking stick or crutch to avoid increasing the burden on allograft. We followed up the patient every 2 months until he or she did not feel pain when walking without a walking stick or crutch.

\section{Statistical methods}

The quantitative data were expressed as the mean \pm SD. Statistical analyses were performed using SPSS version 11 (SPSS Inc., Chicago, IL, USA). The paired $t$ test was used to assess changes in the vertical and horizontal distances before and after revision. A $P$ value of less than 0.05 was considered significant.

\section{Results}

\section{Clinical data}

The HHS improved significantly at the last follow-up compared with the preoperative HHS $(P<0.01)$. The HHS mean was 34 (range, 20-45) preoperatively and 80 (range, 71-98) at the last follow-up. This average is artificially lowered by poor functional scores in some patients due to systemic and concomitant disease in other joints or the loosening of an unrevised femoral component, rather than from an unsatisfactory acetabular reconstruction. At follow-up, none of the components had been re-revised and no deep infections had developed. One intramuscular hematoma required B-ultrasound-guided percutaneous drainage. There were no dislocations in patients. One patient with Paprosky type 3B defects sustained transient femoral and peroneal nerve palsies that resolved without residual weakness or dysesthesia. No patients sustained deep vein thromboses. There were no cases of pulmonary embolism or other systemic complications.

\section{Radiographic data}

As for acetabular migration after operation, the mean postoperative abduction angle of the cage was $49.3^{\circ}$ $\left(34^{\circ}-60^{\circ}\right)$. At the last follow-up, one implant showed more than a $5^{\circ}$ change in the abduction angle, as illustrated in Fig. 1d (right). Restoration of the hip center of 

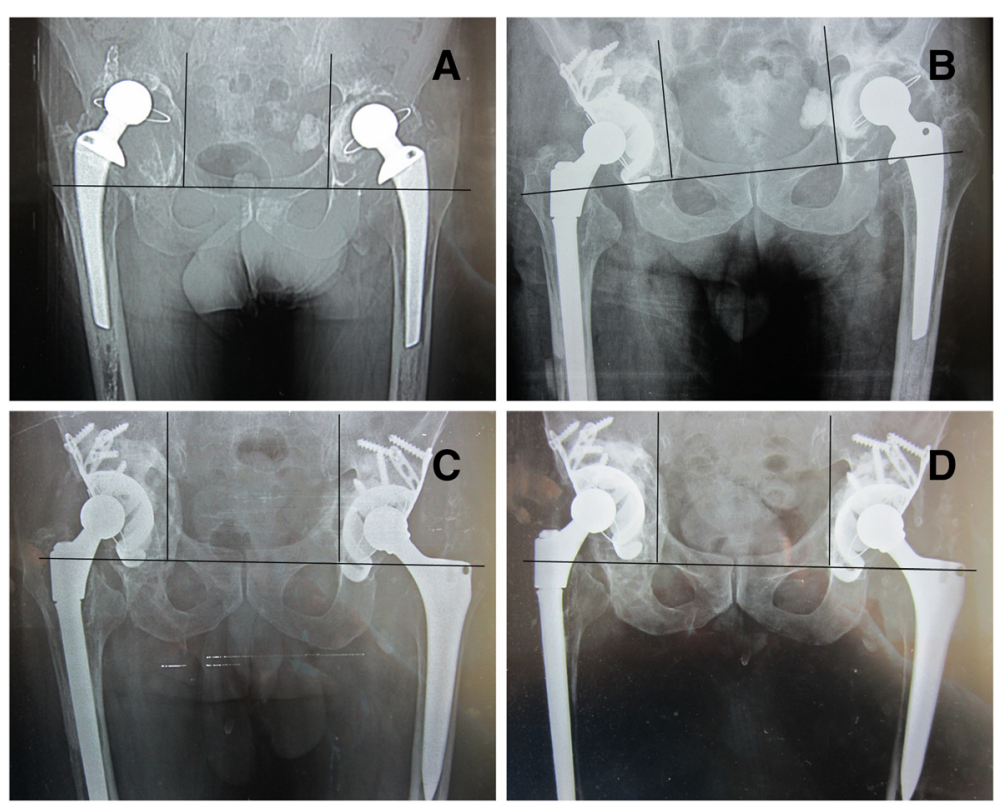

Fig. 1 Preoperative, postoperative, and follow-up radiographic results. a Preoperative radiograph in a 65-year-old man shows massive acetabular bone defects on both sides. $\mathbf{b}$ Immediate postoperative radiograph shows reconstitution with a cage and morselized allograft in the right side. c After 3 months of right revision, the left side also completed the revision. Immediate postoperative radiograph shows reconstitution with a cage and a morselized allograft on the left side. $\mathbf{d}$ Radiograph taken at 7-year follow-up examination indicates that the bone grafts are incorporated relatively incompletely as we can see the sclerotic bone formed at the right side around the cage. And we also can see the acetabular cage move superiorly about $9 \mathrm{~mm}$ and more than a $5^{\circ}$ change in the abduction angle on the right side, but the total reconstruction is still stable. This patient is asymptomatic, and his HSS is 98 at present

rotation was determined by comparing the operated hips pre- and postrevision (Table 1). The horizontal distance averaged $29 \mathrm{~mm}$ preoperatively, which was corrected to $32.9 \mathrm{~mm}$ postoperatively $(P<0.05)$. The vertical distance averaged $36.8 \mathrm{~mm}$ preoperatively, which was corrected to $19.4 \mathrm{~mm}$ postoperatively $(P<0.01)$. On average, the revised hip center of rotation was significantly improved. At the last follow-up, we found that there is no difference between postoperative and last follow-up at horizontal direction $(P>0.05)$. Although there is difference between postoperative and last follow-up at vertical direction $(P<0.05)$, only one hip has more than $5 \mathrm{~mm} \mathrm{mi-}$ gration at the vertical direction, as illustrated in Table 1 (9R) and in Fig. 1d (right). Radiolucencies were present at the bone implant interface in five hips $(17 \%)$. They were all partial radiolucencies, all nonprogressive, and less than $2 \mathrm{~mm}$ in width. There were two radiolucencies in DeLee and Charnley zone II and three in zone III. There were no complete radiolucencies. Incorporation of the allograft was defined as the presence of clearly delineated trabeculae crossing the graft-host junction. This incorporation was complete in 23 hips. Examples are illustrated in Figs. 2 and 3. The six hips with incomplete graft incorporation are illustrated in Fig. 1 (right). No screw, flange, or hook fractures were observed.

\section{Discussion}

Severe pelvic deficiencies present as a difficult problem in hip arthroplasty. Specifically, the goals of restoring or preserving pelvic bone stock, placing the acetabular component in the correct anatomic position, optimizing joint stability, equalizing leg lengths, and achieving stable fixation are not readily achieved in such a situation. Cage reconstruction is an attractive option in that it acts as a plate to bridge the bony defect, protecting the underlying bone graft as it incorporates, and eventually restores pelvic bone stock. This approach allows later revision, if necessary, to be achieved with a regular (uncemented) hemispherical cup.

The midterm result of cage reconstruction with morselized bone allograft is relatively better than other studies using a similar cage construction. Thus far, we have not found any patients in need of re-revision surgery. Although one hip (Fig. 1) shows acetabular migration $>5 \mathrm{~mm}$, which is characterized as definitely loose, and this patient is asymptomatic with an HSS score of 98 at present. This result is comparable to that of Winter et al. [3], who reported no failures among the 38 acetabular reconstructions performed with the Bürch-Schneider cage and morselized allograft at a mean follow-up of 7 years. 
Table 1 Measurement of vertical and horizontal distances of 29 hips at preoperative, immediate postoperative, and final follow-up periods

\begin{tabular}{|c|c|c|c|c|c|c|}
\hline \multirow[b]{2}{*}{ Case no. } & \multicolumn{3}{|c|}{ Horizontal distance (mm) } & \multicolumn{3}{|c|}{ Vertical distance (mm) } \\
\hline & Preoperative & Postoperative & Final & Preoperative & Postoperative & Final \\
\hline 1 & 25.6 & 28.1 & 28.5 & 34 & 23.5 & 26.4 \\
\hline 2 & 26.6 & 28.2 & 28.5 & 26 & 12.8 & 15.4 \\
\hline 3 & 32.7 & 40.5 & 41.3 & 35.4 & 8.5 & 9.6 \\
\hline 4 & 42.5 & 27.8 & 28.4 & 30.5 & 21.5 & 22.5 \\
\hline 5 & 40.5 & 42.9 & 45.7 & 37.1 & 11.1 & 19.2 \\
\hline 6 & 5.2 & 24.9 & 21.2 & 22.2 & 15.3 & 18 \\
\hline 7 & 34.3 & 33.2 & 33.5 & 47.3 & 29.3 & 32.7 \\
\hline 8 & 38.8 & 40.4 & 37.3 & 31.8 & 24 & 26.2 \\
\hline $9 R$ & 32.3 & 43.9 & 39.1 & 58.9 & 22.5 & 33 \\
\hline $9 \mathrm{~L}$ & 30.6 & 30.8 & 30.9 & 40 & 20.8 & 24.7 \\
\hline 10 & 8.5 & 38 & 38.3 & 53.5 & 12.6 & 13 \\
\hline 11 & 19 & 32.6 & 32.3 & 45 & 23.2 & 23.7 \\
\hline 12 & 7.1 & 29.1 & 28.9 & 27.5 & 18.5 & 20.7 \\
\hline 13 & 34 & 36.1 & 35.8 & 34.3 & 17.6 & 18.1 \\
\hline 14 & 31.8 & 30.4 & 31.3 & 29.8 & 16.2 & 18.1 \\
\hline 15 & 38.8 & 40.4 & 37.3 & 31.8 & 24 & 16.2 \\
\hline 16 & 32.3 & 35.3 & 36.2 & 34.7 & 22.8 & 23.4 \\
\hline 17 & 31.5 & 30.2 & 30.1 & 35 & 20.1 & 23.9 \\
\hline 18 & 33.3 & 29 & 31.3 & 43.5 & 25.7 & 26.9 \\
\hline 19 & 34.3 & 32.6 & 31.4 & 38 & 17.8 & 18.9 \\
\hline 20 & 29 & 31.8 & 32.4 & 37.9 & 19.1 & 20.9 \\
\hline 21 & 27.9 & 30.1 & 30.2 & 36.3 & 16.5 & 17.4 \\
\hline 22 & 23.8 & 26.4 & 26.4 & 41.8 & 18.2 & 20 \\
\hline 23 & 25.9 & 27.1 & 28.5 & 37.3 & 23.9 & 24.8 \\
\hline 24 & 31.6 & 32.2 & 32.4 & 30 & 19.8 & 19.4 \\
\hline 25 & 32.7 & 32.5 & 31.6 & 35.9 & 18.3 & 21.6 \\
\hline 26 & 29.9 & 32.8 & 31.9 & 39.5 & 23.5 & 24.9 \\
\hline 27 & 35.5 & 34.2 & 34.1 & 41.1 & 17.3 & 19.9 \\
\hline 28 & 25.2 & 32.8 & 34.2 & 32.2 & 19.3 & 20.1 \\
\hline Average & 29 & 32.9 & 32.7 & 36.8 & 19.4 & 21.6 \\
\hline
\end{tabular}

We believe our success is attributed to the good reconstruction of the host bone, allograft, and cage. The cage provides initial stability, and the allograft incorporation to the host bone provides long-term stability. As for the initial stability, a mechanically stable environment is one of the prerequisites for successful graft incorporation. The cage helps to contain the graft, protect it from overload, and prevent movement between the acetabular component and allograft. In our study, no screw, flange, or hook fractures were observed. This result is likely due to the correct use of the reconstruction cage. In conjunction with the fact that the superior bone stock is very important for a stable fixation of the cage, efforts have been made to achieve close contact between the cage's superior rim and the host bone. Otherwise, migration of the acetabular socket may lead to breakage of the implant. The best choice of cage was the one large enough to directly contact the host bone at the upper side, allowing the inferior hook to be tightly affixed to the teardrop. Attention to these details during the operation can contribute to the early- and long-term stability of total reconstruction.

The incorporation of a bone graft is a dynamic process that involves a common sequence of biologic events: inflammation, revascularization of the bone, resorption of the donor bone, substitution of the graft with new host bone, and remodeling of the construct to provide the required mechanical support to the skeleton [18]. In our 

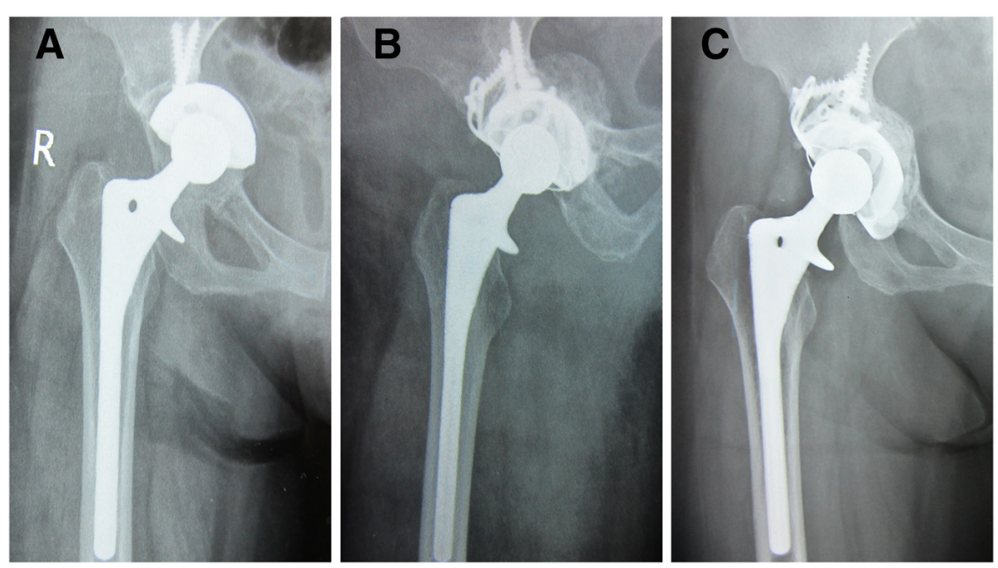

Fig. 2 Bone remodeling after the revision. a Preoperative radiograph of acetabular cup loosening 9 years after primary total hip replacement. b Immediate postoperative radiograph shown with a reconstruction cage and a massive morselized allograft. c Radiograph taken at 8-year follow-up examination indicates bone remodeling has ceased

department, we have three special modifications of this reconstruction technique that we believe are beneficial for bone incorporation. First, we very carefully induced bleeding in the bone bed for the allograft, which is thought to improve revascularization of both the sclerotic bone and graft bone. The upper movement of the cage may have been caused by poor preparation of bone bed on the right side, illustrated in Fig. 1 (right), as the sclerotic bone increased at the last follow-up.

Second, we did not use the impaction technique, as Tägil and Aspenberg [19] demonstrated that the ingrowth of new bone was reduced in impacted grafts in a rat model. Buttaro et al. [5] described a similar reconstruction cage that had failed in 9 of 24 patients with the impacted allograft bone in an average 34-month followup. An additional important reason is that the acetabular cage was stable enough to provide the initial stability without using the impaction technique. Meanwhile, we did not use the structured allograft for the acetabular reconstruction, as we think this may also have reduced the revascularization of allograft. Duffy et al. [4] reported that a similar reconstruction cage had failed in 5 of $12 \mathrm{pa}-$ tients at 5- to 8-year follow-ups with structure allograft.

Third, we did not permit the patient to engage in full weight-bearing activities early in recovery, especially for patients with relatively massive bone defects at the lateral upper region of the acetabulum. Only when the patient felt no pain when walking without a walking stick or crutch did we allow a gradual increase in weightbearing under strict observation at the time of followup. Full weight-bearing should occur only after most of the allograft bone incorporation is complete because we believe early and excessive weight-bearing may damage the total reconstruction; the reasons are as reviewed below. First, there was a large variance in incorporation of the allograft between plain radiograph appearance, other radiographic examination, and the biological and histological observations. The plain radiograph assessment
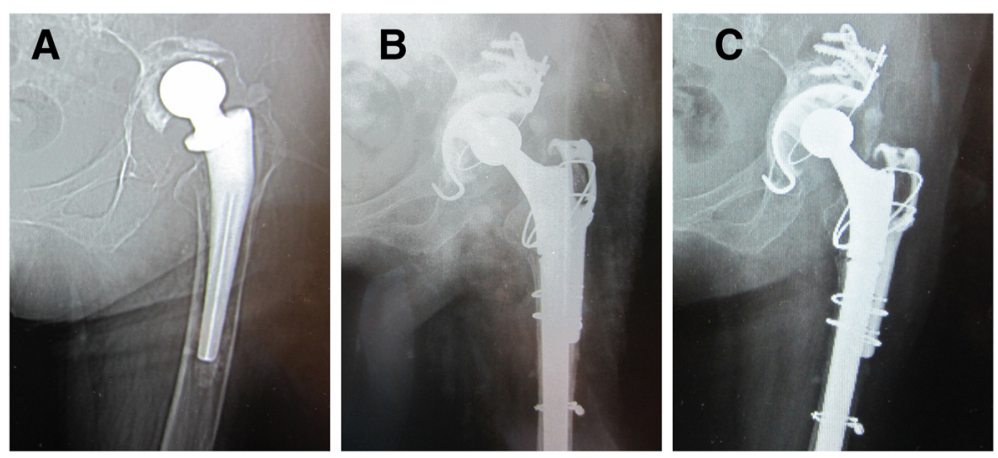

Fig. 3 Preoperative, postoperative, and follow-up radiographs. a Preoperative radiograph of the hip of a 58-year-old woman shows massive acetabular bone defect. $\mathbf{b}$ Immediate postoperative radiograph shows reconstitution with a reconstruction cage and a massive morselized allograft. $\mathbf{c}$ Radiograph taken at 5-year follow-up examination indicates that the cup is stable and the bone graft is incorporated 
tended to overestimate the bone incorporation. One study using single photon emission CT scanning showed the earliest incorporation of cancellous allograft was at 6-18 months [20]. Mall et al. noted only $47 \%$ graft fill and $36 \%$ graft healing in acetabular osteolytic defects following revision using postoperative CT scan analysis at a mean 4.8year follow-up [21]. Core biopsy specimens taken from allograft sites from 20 patients showed allograft remnants remained for up to 18 months after the revision [22]. Although Tägil and Aspenberg [23] suggest that total remodeling of allografts is not necessary because ingrowth of fibrous tissue provides sufficient mechanical properties, we still believe that the long-term stability of the total reconstruction under full weight-bearing conditions requires as much remodeling of the allograft as possible. Second, if performed before the bone incorporation is fully complete, weight-bearing will damage the entire construction. In a study by Buttaro et al. [24] regarding the outcome of larger defects, 6 weeks of unloading with crutches was used, and at 3 years, the survival rate was $90.8 \%$. However, one of the failed results involved a patient who decided to fully load the reconstruction, which resulted in failure. In the Lunn et al. report, we can see that of the 35 revised hips, there were 6 hips with fractured inferior hooks and 2 with screw breakages, which may be caused by early full weight-bearing [25]. Some studies also show that patients at young ages [26] and lower body weights [27] have increased failure rates of revision because these patients are more mobile.

Our study is associated with several notable limitations. First, the absence of a control group makes it difficult to draw conclusions regarding the use of morselized allografts with cemented cage support. Although there are published data that serve as a historical control, it is not possible to adjust for confounding between studies. Second, the minimum follow-up of 4 years does not allow accurate assessment of the long-term efficacy of the technique. Third, incorporation of the allograft was only evaluated on radiograph. Although some authors [28] developed the grading system of allograft appearance with more detailed means, they still use the radiograph to assess the graft incorporation. Just like Zehntner [15] described in his research, incorporation of the central portions of the graft was not assessed since the fate of these parts is still unclear, and there seems to be evidence that they are not revascularized with reliable probability. So CT scan of the hip is probably a good solution to define incorporation despite possible metal artifacts.

\section{Conclusion}

Technical decisions during surgery can prolong the durability of acetabular reconstructions with morselized allografts and cemented reconstruction cages. On the one hand, the correct fixation of the reconstruction cage is important for providing initial stability. On the other hand, we aimed to improve the revascularization of the allograft bone (not using the impaction technique or structure allograft) and the interface between the allograft and host bone (by preparing the bleeding bone bed), which may increase the allograft incorporation. Moreover, avoiding weight-bearing activity too early during recovery with strict, on-time follow-ups before the total bone incorporation may also contribute to the success of the reconstruction. In conclusion, reconstructions consisting of a morselized allograft without impaction and cemented cage are a good option for massive bone deficiencies in developing country, if used properly.

\section{Competing interests}

The authors declare that they have no competing interests.

\section{Authors' contributions}

$B Y$ and $Z Z$ conceived the study. $H D, B Y, Y M, B Y, H L$, and $Z Z$ performed the surgery and followed up the patients. $\mathrm{HFD}, \mathrm{BY}$, and $\mathrm{JH}$ analyzed the data. All authors were involved in writing the paper and had final approval of the submitted and published version.

\section{Acknowledgements}

This work was supported by the Program for the Shanghai Key Laboratory of Orthopaedic Implant (Grant No. 08DZ2230300).

Received: 9 February 2015 Accepted: 10 May 2015

Published online: 23 May 2015

\section{References}

1. Berry DJ, Muller ME. Revision arthroplasty using an anti-protrusio cage for massive acetabular bone deficiency. J Bone Joint Surg (Br). 1992;74:711-5.

2. Gill TJ, Sledge JB, Muller ME. The Burch-Schneider anti-protrusio cage in revision total hip arthroplasty: indications, principles and long-term results. J Bone Joint Surg (Br). 1998;80:946-53.

3. Winter E, Piert M, Volkmann R, Maurer F, Eingartner C, Weise K, et al. Allogeneic cancellous bone graft and a Burch-Schneider ring for acetabular reconstruction in revision hip arthroplasty. J Bone Joint Surg Am. 2001;83-A:862-7.

4. Duffy GP, O'Connor MI, Brodersen MP. Fatigue failure of the GAP ring. J Arthroplasty. 2007;22:711-4.

5. Buttaro MA, de la Rosa DM, Comba F, Piccaluga F. High failure rate with the GAP I| ring and impacted allograft bone in severe acetabular defects. Clin Orthop Relat Res. 2012;470:3148-55.

6. Böhm P, Banzhaf S. Acetabular revision with allograft bone. 103 revisions with 3 reconstruction alternatives, followed for 0.3-13 years. Acta Orthop Scand. 1999;70:240-9.

7. Sembrano JN, Cheng EY. Acetabular cage survival and analysis of factors related to failure. Clin Orthop Relat Res. 2008;466:1657-65.

8. Sun C, Lian YY, Jin YH, Zhao CB, Pan SQ, Liu XF. Clinical and radiographic assessment of cementless acetabular revision with morsellised allografts. Int Orthop. 2009;33:1525-30.

9. Paprosky WG, Perona PG, Lawrence JM. Acetabular defect classification and surgical reconstruction in revision arthroplasty. A 6-year follow-up evaluation. J Arthroplasty. 1994;9:33-44.

10. Harris WH. Traumatic arthritis of the hip after dislocation and acetabular fractures: treatment by mold arthroplasty. An end-result study using a new method of result evaluation. J Bone Joint Surg Am. 1969;51:737-55.

11. Peters $C L$, Curtain M, Samuelson KM. Acetabular revision with the Burch-Schnieder antiprotrusio cage and cancellous allograft bone. J Arthroplasty. 1995;10:307-12.

12. DeLee JG, Charnley J. Radiological demarcation of cemented sockets in total hip replacement. Clin Orthop Relat Res. 1976;121:20-32.

13. Gill TJ, Sledge JB, Muller ME. The management of severe acetabular bone loss using structural allograft and acetabular reinforcement devices. J Arthroplast. 2000;15:1-7. 
14. van der Linde M, Tonino A. Acetabular revision with impacted grafting and a reinforcement ring: 42 patients followed for a mean of 10 years. Acta Orthop Scand. 2001;72:221-7.

15. Zehntner MK, Ganz R. Midterm results (5.5-10 years) of acetabular allograft reconstruction with the acetabular reinforcement ring during total hip revision. J Arthroplasty. 1994;9:469-79.

16. Udomkiat $P$, Dorr LD, Won YY, Longjohn D, Wan Z. Technical factors for success with metal ring acetabular reconstruction. J Arthroplasty. 2001;16:961-9.

17. Kim KC, Ha YC, Kang BJ, Lee YK, Ji HM, Koo KH. Use of cementless acetabular component with a hook and iliac flanges in revision arthroplasty for massive acetabular defect. J Orthop Sci. 2012;17:18-24.

18. Goldberg VM, Stevenson S. The biology of bone grafts. Semin Arthroplasty. 1993;4:58-63.

19. Tägil M, Aspenberg P. Impaction of cancellous bone grafts impairs osteoconduction in titanium chambers. Clin Orthop Relat Res. 1998;352:231-8.

20. Shih $\mathrm{CH}$, Chen $\mathrm{CH}$, Tsai MF, Tzen KY. Incorporation of allograft for acetabular reconstruction. Single photon emission CT in 21 hip arthroplasties followed for 2.5-5 years. Acta Orthop Scand. 1994;65:589-94.

21. Mall NA, Nunley RM, Smith KE, Maloney WJ, Clohisy JC, Barrack RL. The fate of grafting acetabular defects during revision total hip arthroplasty. Clin Orthop Relat Res. 2010;468:3286-94.

22. Heekin RD, Engh CA, Vinh T. Morselized allograft in acetabular reconstruction. A postmortem retrieval analysis. Clin Orthop Relat Res. 1995;319:184-90.

23. Tägil M, Aspenberg P. Fibrous tissue armoring increases the mechanical strength of an impacted bone graft. Acta Orthop Scand. 2001;72:78-82.

24. Buttaro MA, Comba F, Pusso R, Piccaluga F. Acetabular revision with metal mesh, impaction bone grafting, and a cemented cup. Clin Orthop Relat Res. 2008;466:2482-90.

25. Lunn JV, Kearns SS, Quinlan W, Murray P, Byrne JO. Impaction allografting and the Kerboull acetabular reinforcement device: 35 hips followed for 3-7 years. Acta Orthop. 2005;76:296-302.

26. Schreurs BW, Slooff TJ, Gardeniers JW, Buma P. Acetabular reconstruction with bone impaction grafting and a cemented cup: 20 years' experience. Clin Orthop Relat Res. 2001;393:202-15.

27. Nivbrant B, Karrholm J. Migration and wear of hydroxyapatite-coated press-fit cups in revision hip arthroplasty: a radiostereometric study. J Arthroplasty. 1997;12:904-12.

28. Gie GA, Linder L, Ling RS, Simon JP, Slooff TJ, Timperley AJ. Impacted cancellous allografts and cement for revision total hip arthroplasty. J Bone Joint Surg (Br). 1993;75:14-21.

\section{Submit your next manuscript to BioMed Central and take full advantage of:}

- Convenient online submission

- Thorough peer review

- No space constraints or color figure charges

- Immediate publication on acceptance

- Inclusion in PubMed, CAS, Scopus and Google Scholar

- Research which is freely available for redistribution 